\title{
RAINBOW DISCONNECTION IN GRAPHS
}

\author{
Gary Chartrand ${ }^{1}$, Stephen Devereaux ${ }^{2}$, Teresa W. Haynes ${ }^{3}$, \\ Stephen T. Hedetniemi ${ }^{4}$ and Ping Zhang $^{1}$ \\ ${ }^{1}$ Department of Mathematics \\ Western Michigan University \\ Kalamazoo, MI 49008-5248 USA \\ ${ }^{2}$ Department of Mathematics \\ Cornerstone University \\ Grand Rapids, MI 49525 USA \\ ${ }^{3}$ Department of Mathematics and Statistics \\ East Tennessee State University \\ Johnson City, TN 37614-0002 USA \\ ${ }^{4}$ School of Computing \\ Clemson University \\ Clemson, SC 29634 USA \\ e-mail: gary.chartrand@wmich.edu \\ stephen.devereaux@cornerstone.edu \\ haynes@etsu.edu \\ hedet@clemson.edu \\ ping.zhang@wmich.edu
}

\begin{abstract}
Let $G$ be a nontrivial connected, edge-colored graph. An edge-cut $R$ of $G$ is called a rainbow cut if no two edges in $R$ are colored the same. An edgecoloring of $G$ is a rainbow disconnection coloring if for every two distinct vertices $u$ and $v$ of $G$, there exists a rainbow cut in $G$, where $u$ and $v$ belong to different components of $G-R$. We introduce and study the rainbow disconnection number $\operatorname{rd}(G)$ of $G$, which is defined as the minimum number of colors required of a rainbow disconnection coloring of $G$. It is shown that the rainbow disconnection number of a nontrivial connected graph $\mathrm{G}$ equals the maximum rainbow disconnection number among the blocks of $\mathrm{G}$. It is also shown that for a nontrivial connected graph $G$ of order $n, \operatorname{rd}(G)=n-1$ if and only if $G$ contains at least two vertices of degree $n-1$. The rainbow disconnection numbers of all grids $P_{m} \square P_{n}$ are determined. Furthermore, it is shown for integers $k$ and $n$ with $1 \leq k \leq n-1$ that the minimum
\end{abstract}


size of a connected graph of order $n$ having rainbow disconnection number $k$ is $n+k-2$. Other results and a conjecture are also presented.

Keywords: edge coloring, rainbow connection, rainbow disconnection.

2010 Mathematics Subject Classification: 05C15, 05C40.

\section{INTRODUCTION}

An edge-coloring of a graph $G$ is a function $c: E(G) \rightarrow[k]=\{1,2, \ldots, k\}$ for some positive integer $k$ where adjacent edges may be assigned the same color. A graph with an edge-coloring is an edge-colored graph. If no two adjacent edges of $G$ are colored the same, then $c$ is a proper edge-coloring. The minimum number of colors required of a proper edge-coloring of $G$ is the chromatic index of $G$, denoted by $\chi^{\prime}(G)$. The minimum and maximum degrees of $G$ are denoted by $\delta(G)$ and $\Delta(G)$, respectively. By a famous 1964 theorem of Vizing [7],

$$
\Delta(G) \leq \chi^{\prime}(G) \leq \Delta(G)+1
$$

for every nonempty graph $G$.

A set $R$ of edges in a connected edge-colored graph $G$ is a rainbow set if no two edges in $R$ are colored the same. A path $P$ in $G$ is a rainbow path if no two edges in $P$ are colored the same. The graph $G$ is rainbow-connected if every two vertices of $G$ are connected by a rainbow path. An edge-coloring of $G$ with this property is called a rainbow coloring. The minimum number of colors needed in a rainbow coloring of $G$ is the rainbow connection number of $G$, denoted by $\operatorname{rc}(G)$. Rainbow connection was introduced [1] in 2006. For more details on rainbow connection, see the book [6] and the survey paper[5].

The object of this paper is to introduce a concept that is somewhat reverse to rainbow connection and to present some results dealing with this new concept.

\section{An Introduction to Rainbow Disconnection}

An edge-cut of a nontrivial connected graph $G$ is a set $R$ of edges of $G$ such that $G-R$ is disconnected. The minimum number of edges in an edge-cut of $G$ is its edge-connectivity $\lambda(G)$. We then have the well-known inequality $\lambda(G) \leq \delta(G)$. For two distinct vertices $u$ and $v$ of $G$, let $\lambda(u, v)$ denote the minimum number of edges in an edge-cut $R$ of $G$ such that $u$ and $v$ lie in different components of $G-R$. The following result of Elias, Feinstein and Shannon [2] and Ford and Fulkerson [3] presents an alternate interpretation of $\lambda(u, v)$.

Theorem 2.1. For every two vertices $u$ and $v$ in a graph $G, \lambda(u, v)$ is the maximum number of pairwise edge-disjoint $u-v$ paths in $G$. 
The upper edge-connectivity $\lambda^{+}(G)$ is defined by

$$
\lambda^{+}(G)=\max \{\lambda(u, v): u, v \in V(G)\} .
$$

Consider, for example, the graph $K_{n}+v$ obtained from the complete graph $K_{n}$, one vertex of which is attached to a single leaf $v$. For this graph, $\lambda\left(K_{n}+\right.$ $v)=1$ while $\lambda^{+}\left(K_{n}+v\right)=n-1$. Thus, $\lambda(G)$ denotes the global minimum edge-connectivity of a graph, while $\lambda^{+}(G)$ denotes the local maximum edgeconnectivity of a graph.

A set $R$ of edges in a nontrivial connected, edge-colored graph $G$ is a rainbow cut of $G$ if $R$ is both a rainbow set and an edge-cut. A rainbow cut $R$ is said to separate two vertices $u$ and $v$ of $G$ if $u$ and $v$ belong to different components of $G-R$. Any such rainbow cut in $G$ is called a $u-v$ rainbow cut in $G$. An edge-coloring of $G$ is a rainbow disconnection coloring if for every two distinct vertices $u$ and $v$ of $G$, there exists a $u-v$ rainbow cut in $G$. The rainbow disconnection number $\operatorname{rd}(G)$ of $G$ is the minimum number of colors required of a rainbow disconnection coloring of $G$. A rainbow disconnection coloring with $\operatorname{rd}(G)$ colors is called an rd-coloring of $G$. We now present bounds for the rainbow disconnection number of a graph.

Proposition 2.2. If $G$ is a nontrivial connected graph, then

$$
\lambda(G) \leq \lambda^{+}(G) \leq \operatorname{rd}(G) \leq \chi^{\prime}(G) \leq \Delta(G)+1 .
$$

Proof. First, by Vizing's theorem, $\chi^{\prime}(G) \leq \Delta(G)+1$. Now, let there be given a proper edge-coloring of $G$ using $\chi^{\prime}(G)$ colors. Then, for each vertex $x$ of $G$, the set $E_{x}$ of edges incident with $x$ is a rainbow set and $\left|E_{x}\right|=\operatorname{deg} x \leq \Delta(G) \leq \chi^{\prime}(G)$. Furthermore, $E_{x}$ is a rainbow cut in $G$ and so $\operatorname{rd}(G) \leq \chi^{\prime}(G)$.

Next, let there be given an rd-coloring of $G$. Let $u$ and $v$ be two vertices of $G$ such that $\lambda^{+}(G)=\lambda(u, v)$ and let $R$ be a $u-v$ rainbow cut with $|R|=\lambda(u, v)$. Then $|R| \leq \operatorname{rd}(G)$. Thus, $\lambda(G) \leq \lambda^{+}(G)=|R| \leq \operatorname{rd}(G)$.

We now present examples of two classes of connected graphs $G$ for which $\lambda(G)=\operatorname{rd}(G)$, namely cycles and wheels.

Proposition 2.3. If $C_{n}$ is a cycle of order $n \geq 3$, then $\operatorname{rd}\left(C_{n}\right)=2$.

Proof. Since $\lambda\left(C_{n}\right)=2$, it follows by Proposition 2.2 that $\operatorname{rd}\left(C_{n}\right) \geq 2$. To show that $\operatorname{rd}\left(C_{n}\right) \leq 2$, let $c$ be an edge-coloring of $C_{n}$ that assigns the color 1 to exactly $n-1$ edges of $C_{n}$ and the color 2 to the remaining edge $e$ of $C_{n}$. Let $u$ and $v$ be two vertices of $C_{n}$. There are two $u-v$ paths $P$ and $Q$ in $C_{n}$, exactly one of which contains the edge $e$, say $e \in E(P)$. Then any set $\{e, f\}$, where $f \in E(Q)$, is a $u-v$ rainbow cut. Thus, $c$ is a rainbow disconnection coloring of $C_{n}$ using two colors. Hence, $\operatorname{rd}\left(C_{n}\right)=2$. 
Proposition 2.4. If $W_{n}=C_{n} \vee K_{1}$ is the wheel of order $n+1 \geq 4$, then $\operatorname{rd}\left(W_{n}\right)=3$.

Proof. Since $\lambda\left(W_{n}\right)=3$, it follows by Proposition 2.2 that $\operatorname{rd}\left(W_{n}\right) \geq 3$. It remains to show that there is a rainbow disconnection coloring of $W_{n}$ using only the colors 1,2,3. Suppose that $C_{n}=\left(v_{1}, v_{2}, \ldots, v_{n}, v_{1}\right)$ and that $v$ is the center of $W_{n}$. Define an edge-coloring $c: E\left(W_{n}\right) \rightarrow\{1,2,3\}$ of $W_{n}$ as follows. First, let $c$ be a proper edge-coloring of $C_{n}$ using the colors 1,2,3. For each integer $i$ with $1 \leq i \leq n$, let $a_{i} \in\{1,2,3\}-\left\{c\left(v_{i-1} v_{i}\right), c\left(v_{i} v_{i+1}\right)\right\}$ where each subscript is expressed as an integer $1,2, \ldots, n$ modulo $n$, and let $c\left(v v_{i}\right)=a_{i}$. Thus, the set $E_{v_{i}}$ of the three edges incident with $v_{i}$ is a rainbow set for $1 \leq i \leq n$. Let $x$ and $y$ be two distinct vertices of $W_{n}$. Then at least one of $x$ and $y$ belongs to $C_{n}$, say $x \in V\left(C_{n}\right)$. Since $E_{x}$ separates $x$ and $y$, it follows that $c$ is a rainbow disconnection coloring of $W_{n}$ using three colors. Hence, $\operatorname{rd}\left(W_{n}\right)=3$.

Since $\chi^{\prime}\left(C_{n}\right)=3$ when $n \geq 3$ is odd and $\chi^{\prime}\left(W_{n}\right)=n$ for each integer $n \geq 3$, it follows that $\operatorname{rd}(G)<\chi^{\prime}(G)$ if $G$ is an odd cycle or if $G$ is a wheel of order at least 4 . Wheels therefore illustrate that there are graphs $G$ for which $\chi^{\prime}(G)-\operatorname{rd}(G)$ can be arbitrarily large. We now give an example of a graph $G$ for which $\lambda^{+}(G)<\operatorname{rd}(G)=\chi^{\prime}(G)$.

Proposition 2.5. The rainbow disconnection number of the Petersen graph is 4 .

Proof. Let $P$ denote the Petersen graph where $V(P)=\left\{v_{1}, v_{2}, \ldots, v_{10}\right\}$. Since $\lambda(P)=3$ and $\chi^{\prime}(P)=4$, it follows by Proposition 2.2 that $\operatorname{rd}(P)=3$ or $\operatorname{rd}(P)=$ 4. Assume, to the contrary, that $\operatorname{rd}(P)=3$ and let there be given a rainbow disconnection 3-coloring of $P$. Now, let $u$ and $v$ be two vertices of $P$ and let $R$ be a $u-v$ rainbow cut. Hence, $|R| \leq 3$ and $P-R$ is disconnected, where $u$ and $v$ belong to different components of $P-R$. Let $U$ be the vertex set of the component of $P-R$ containing $u$, where $|U|=k$. We may assume that $1 \leq k \leq 5$. First, suppose that $1 \leq k \leq 4$. Since the girth of $P$ is 5 , the subgraph $P[U]$ induced by $U$ contains $k-1$ edges. Therefore, $|R|=3 k-(2 k-2)=k+2$, where then $3 \leq k+2 \leq 6$. If $k=5$, then $P[U]$ contains at most five edges and so $|R| \geq 5$, which is impossible. Since $\operatorname{rd}(P)=3$, it follows that $|R| \leq 3$ and so $k=1$. Hence, the only possible $u-v$ rainbow cut is the set of the three edges incident with $u$ (or with $v$ ).

Let the colors assigned to the edges of $P$ be red, blue and green. Since $\chi^{\prime}(P)=4$, there is at least one vertex of $P$ that is incident with two edges of the same color. We claim, in fact, that there are at least two such vertices. Let $E_{R}$, $E_{B}$ and $E_{G}$ denote the sets of edges of $P$ colored red, blue and green, respectively, and let $P_{R}, P_{B}$ and $P_{G}$ be the spanning subgraphs of $P$ with edge sets $E_{R}, E_{B}$ and $E_{G}$. We may assume that $\left|E_{R}\right| \geq\left|E_{B}\right| \geq\left|E_{G}\right|$ and so $\left|E_{R}\right| \geq 5$. If $\left|E_{R}\right| \geq 7$, then $\sum_{i=1}^{10} \operatorname{deg}_{P_{R}} v_{i} \geq 14$. Since $\operatorname{deg}_{P_{R}} v_{i} \leq 3$ for each $i$ with $1 \leq i \leq 10$, at least 
two vertices are incident with two red edges, verifying the claim. If $\left|E_{R}\right|=6$, then $\sum_{i=1}^{10} \operatorname{deg}_{P_{R}} v_{i}=12$. Then either (i) at least two vertices are incident with two red edges or (ii) there is a vertex, say $v_{10}$, incident with three red edges and each of $v_{1}, v_{2}, \ldots, v_{9}$ is incident with exactly one red edge. If (ii) occurs, then either $\left|E_{B}\right|=6$ or $\left|E_{B}\right|=5$ and so $\sum_{i=1}^{9} \operatorname{deg}_{P_{B}} v_{i} \geq 10$, which implies that at least one of the vertices $v_{1}, v_{2}, \ldots, v_{9}$ is incident with two blue edges, again verifying the claim.

The only remaining possibility is therefore $\left|E_{R}\right|=\left|E_{B}\right|=\left|E_{G}\right|=5$. If $E_{R}$ is an independent set of five edges, then $P-E_{R}$ is a 2-regular graph. Since the girth of $P$ is 5 and $P$ is not Hamiltonian, it follows that $P-E_{R}$ consists of two vertex-disjoint 5 -cycles. Thus, there is a vertex of $P$ in each cycle incident with two blue edges or with two green edges, verifying the claim. Hence, none of $E_{R}, E_{B}$ or $E_{G}$ is an independent set. This implies that for each of these colors, there is a vertex of $P$ incident with two edges of this color, verifying the claim in general.

Thus, $P$ contains two vertices $u$ and $v$, each of which is incident with two edges of the same color. Since the only $u-v$ rainbow cut is the set of edges incident with $u$ or $v$, this is a contradiction.

The following two results are useful.

Proposition 2.6. If $H$ is a connected subgraph of a graph $G$, then $\operatorname{rd}(H) \leq$ $\operatorname{rd}(G)$.

Proof. Let $c$ be an rd-coloring of $G$ and let $u$ and $v$ are two vertices of $G$. Suppose that $R$ is a $u-v$ rainbow cut. Then $R \cap E(H)$ is a $u-v$ rainbow cut in $H$. Hence, $c$ restricted to $H$ is a rainbow disconnection coloring of $H$. Thus, $\operatorname{rd}(H) \leq \operatorname{rd}(G)$.

A block of a graph is a maximal connected graph of $G$ containing no cutvertices. The block decomposition of $G$ is the set of blocks of $G$.

Proposition 2.7. Let $G$ be a nontrivial connected graph, and let $B$ be a block of $G$ such that $\operatorname{rd}(B)$ is maximum among all blocks of $G$. Then $\operatorname{rd}(G)=\operatorname{rd}(B)$.

Proof. Let $G$ be a nontrivial connected graph. Let $\left\{B_{1}, B_{2}, \ldots, B_{t}\right\}$ be a block decomposition of $G$, and let $k=\max \left\{\operatorname{rd}\left(B_{i}\right) \mid 1 \leq i \leq t\right\}$. If $G$ has no cut-vertex, then $G=B_{1}$ and the result follows. Hence, we may assume that $G$ has at least one cutvertex. By Proposition 2.6, $k \leq \operatorname{rd}(G)$.

Let $c_{i}$ be an rd-coloring of $B_{i}$. We define the edge-coloring $c: E(G) \rightarrow[k]$ of $G$ by $c(e)=c_{i}(e)$ if $e \in E\left(B_{i}\right)$.

Let $x, y \in V(G)$. If there exists a block, say $B_{i}$, that contains both $x$ and $y$, then any $x-y$ rainbow cut in $B_{i}$ is an $x-y$ rainbow cut in $G$. Hence, we can assume that no block of $G$ contains both $x$ and $y$, and that $x \in B_{i}$ and $y \in B_{j}$, 
where $i \neq j$. Now every $x-y$ path contains a cut-vertex, say $v$, of $G$ in $B_{i}$ and a cutvertex, say $w$, of $G$ in $B_{j}$. Note that $v$ could equal $w$. If $x \neq v$, then any $x-v$ rainbow cut of $B_{i}$ is an $x-y$ rainbow cut in $G$. Similarly, if $y \neq w$, then any $y-w$ rainbow cut of $B_{j}$ is an $x-y$ rainbow cut in $G$. Thus, we may assume that $x=v$ and $y=w$. It follows that $v \neq w$. Consider the $x-y$ path $P=\left(x=v_{1}, v_{2}, \ldots, v_{p}=y\right)$. Since $x$ and $y$ are cutvertices in different blocks and no block contains both $x$ and $y, P$ contains a cut-vertex $z$ of $G$ in $B_{i}$, that is, $z=v_{k}$ for some $k(2 \leq k \leq p-1)$. Then any $x-z$ rainbow cut of $B_{i}$ is an $x-y$ rainbow cut of $G$. Hence, $\operatorname{rd}(G) \leq k$, and so $\operatorname{rd}(G)=k$.

As a consequence of Proposition 2.7, the study of rainbow disconnection numbers can be restricted to 2 -connected graphs. We now present several corollaries of Proposition 2.7.

Corollary 2.8. Let $G$ and $H$ be any two nontrivial connected graphs, and let $G v H$ be a graph formed by identifying a vertex in $G$ with a vertex in $H$. Then $\operatorname{rd}(G v H)=\max \{\operatorname{rd}(G), \operatorname{rd}(H)\}$.

Corollary 2.9. Let $G$ and $H$ be any two nontrivial connected graphs, and let GuvH be a graph formed by adding an edge between any vertex $u$ in $G$ and any vertex $v$ in $H$. Then $\operatorname{rd}(G u v H)=\max \{\operatorname{rd}(G), \operatorname{rd}(H)\}$.

Corollary 2.10. Let $G$ be a nontrivial connected graph and $G^{\prime}$ the graph obtained by attaching a pendant edge uv to some vertex $u$ of $G$. Then $\operatorname{rd}\left(G^{\prime}\right)=\operatorname{rd}(G)$.

The corona $G \circ K_{1}$ is the graph obtained from $G$ by attaching a leaf to each vertex of $G$. Thus, if $G$ has order $n$, then the corona $G \circ K_{1}$ has order $2 n$ and has precisely $n$ leaves.

Corollary 2.11. If $G$ is a nontrivial connected graph, then $\operatorname{rd}\left(G \circ K_{1}\right)=\operatorname{rd}(G)$.

Corollary 2.12. Let $G$ be a nontrivial connected graph, let $T$ be a nontrivial tree and let $u$ and $v$ be vertices of $G$ and $T$, respectively. If $H$ is the graph obtained from $G$ and $T$ by identifying $u$ and $v$, then $\operatorname{rd}(H)=\operatorname{rd}(G)$.

A unicyclic graph is a connected graph with exactly one cycle.

Corollary 2.13. If $G$ is a unicyclic graph $G$, then $\operatorname{rd}(G)=2$.

\section{Graphs with Prescribed Order and Rainbow Disconnection NUMBER}

In this section, we characterize all those nontrivial connected graphs of order $n$ with rainbow disconnection number $k$ for each $k \in\{1,2, n-1\}$. The result for graphs having rainbow disconnection number 1 follows directly from Propositions 2.6 and 2.7 . 
Proposition 3.1. Let $G$ be a nontrivial connected graph. Then $\operatorname{rd}(G)=1$ if and only if $G$ is a tree.

Next, we characterize all nontrivial connected graphs of order $n$ having rainbow disconnection number 2. By Proposition 3.1, such a graph must contain a cycle. An ear of a graph $G$ is a maximal path whose internal vertices have degree 2 in $G$. An ear decomposition of a graph is a decomposition $H_{0}, H_{1}, \ldots, H_{k}$ such that $H_{0}$ is a cycle in $G$ and $H_{i}$ is an ear of the subgraph of $G$ with edge set $E\left(H_{0}\right) \cup E\left(H_{1}\right) \cup \cdots \cup E\left(H_{i}\right)$ for each integer $i$ with $1 \leq i \leq k$. Whitney [8] proved the following result in 1932.

Theorem 3.2. A graph $G$ is 2-connected if and only if $G$ has an ear decomposition. Furthermore, every cycle is the initial cycle in some ear decomposition of $G$.

The following is a consequence of Theorem 3.2.

Lemma 3.3. A 2-connected graph $G$ is a cycle if and only if for every two vertices $u$ and $v$ of $G$, there are exactly two internally disjoint $u-v$ paths in $G$.

Also, by Theorem 3.2, if $G$ is a 2-connected, non-Hamiltonian graph, then $G$ contains a theta subgraph (a subgraph consisting of two vertices connected by three internally disjoint paths of length 2 or more).

Theorem 3.4. Let $G$ be a nontrivial connected graph. Then $\operatorname{rd}(G)=2$ if and only if each block of $G$ is either $K_{2}$ or a cycle and at least one block of $G$ is a cycle.

Proof. If $G$ a nontrivial connected graph, each block of which is either $K_{2}$ or a cycle and at least one block of $G$ is a cycle, then Propositions 2.3 and 2.7 imply that $\operatorname{rd}(G)=2$.

We now verify the converse. Assume, to the contrary, that there is a connected graph $G$ with $\operatorname{rd}(G)=2$ that does not have the property that each block of $G$ is either $K_{2}$ or a cycle and at least one block of $G$ is a cycle. First, not all blocks can be $K_{2}$, for otherwise, $G$ is a tree and so $\operatorname{rd}(G)=1$ by Proposition 3.1. Hence, $G$ contains a block that is neither $K_{2}$ nor a cycle. By Lemma 3.3, there exist two distinct vertices $u$ and $v$ of $G$ for which $G$ contains at least three internally disjoint $u-v$ paths $P_{1}, P_{2}$ and $P_{3}$. Thus, any $u-v$ rainbow cut $R$ must contain at least one edge from each of $P_{1}, P_{2}$ and $P_{3}$ and so $|R| \geq 3$, which is impossible.

We now consider those graphs that are, in a sense, opposite to trees.

Proposition 3.5. For each integer $n \geq 4, \operatorname{rd}\left(K_{n}\right)=n-1$. 
Proof. Suppose first that $n \geq 4$ is even. Then $\lambda\left(K_{n}\right)=\chi^{\prime}\left(K_{n}\right)=n-1$. It then follows by Proposition 2.2 that $\operatorname{rd}\left(K_{n}\right)=n-1$. Next, suppose that $n \geq 5$ is odd. Then $n-1=\lambda\left(K_{n}\right) \leq \operatorname{rd}\left(K_{n}\right) \leq \chi^{\prime}\left(K_{n}\right)=n$ by Proposition 2.2. To show that $\operatorname{rd}\left(K_{n}\right)=n-1$, it remains to show that there is a rainbow disconnection coloring of $K_{n}$ using $n-1$ colors. Let $x \in V\left(K_{n}\right)$. Then $K_{n}-x=K_{n-1}$. Since $n-1$ is even, it follows that $\chi^{\prime}\left(K_{n-1}\right)=n-2$. Thus, there is a proper edge-coloring $c_{0}$ of $K_{n-1}$ using the colors $1,2, \ldots, n-2$. We now extend $c_{0}$ to an edge-coloring $c$ of $K_{n}$ by assigning the color $n-1$ to each edge of $K_{n}$ that is incident with $x$. We show that $c$ is a rainbow disconnection coloring of $K_{n}$. Let $u$ and $v$ be two vertices of $K_{n}$, where say $u \neq x$. Then the set $E_{u}$ of edges incident with $u$ is a $u-v$ rainbow cut. Thus, $c$ is a rainbow disconnection coloring of $K_{n}$ and so $\operatorname{rd}\left(K_{n}\right) \leq n-1$ and so $\operatorname{rd}\left(K_{n}\right)=n-1$.

By Propositions 2.2, 2.6 and 3.5, if $G$ is a nontrivial connected graph of order $n$, then

$$
1 \leq \operatorname{rd}(G) \leq n-1
$$

Furthermore, $\operatorname{rd}(G)=1$ if and only if $G$ is a tree by Proposition 3.1. We have seen that the complete graphs $K_{n}$ of order $n \geq 2$ have rainbow disconnection number $n-1$. We now characterize all nontrivial connected graphs of order $n$ having rainbow disconnection number $n-1$.

Theorem 3.6. Let $G$ be a nontrivial connected graph of order $n$. Then $\operatorname{rd}(G)=$ $n-1$ if and only if $G$ contains at least two vertices of degree $n-1$.

Proof. First, suppose that $G$ is a nontrivial connected graph of order $n$ containing at least two vertices of degree $n-1$. Since $\operatorname{rd}(G) \leq n-1$ by (1), it remains to show that $\operatorname{rd}(G) \geq n-1$. Let $u, v \in V(G)$ such that $\operatorname{deg} u=\operatorname{deg} v=n-1$. Among all sets of edges that separate $u$ and $v$ in $G$, let $S$ be one of minimum size. We show that $|S| \geq n-1$. Let $U$ be a component of $G-S$ that contains $u$ and let $W=V(G)-U$. Thus, $v \in W$ and $S=[U, W]$ consists of those edges in $G-S$ joining a vertex of $U$ and a vertex of $W$. Suppose that $|U|=k$ for some integer $k$ with $1 \leq k \leq n-1$ and then $|W|=n-k$. The vertex $u$ is adjacent to each of the $n-k$ vertices of $W$ and each of the remaining $k-1$ vertices in $U$ is adjacent to at least one vertex in $W$. Hence, $|S| \geq n-k+(k-1)=n-1$. This implies that every $u-v$ rainbow cut contains at least $n-1$ edges of $G$ and so $\operatorname{rd}(G) \geq n-1$.

For the converse, suppose that $G$ is a nontrivial connected graph of order $n$ having at most one vertex of degree $n-1$. We show that $\operatorname{rd}(G) \leq n-2$. We consider two cases.

Case 1. Exactly one vertex $v$ of $G$ has degree $n-1$. Let $H=G-v$. Thus, $\Delta(H) \leq n-3$. Since $\chi^{\prime}(H) \leq \Delta(H)+1=n-2$, there is a proper edge-coloring 
of $H$ using $n-2$ colors. We now define an edge-coloring $c: E(G) \rightarrow[n-2]$ of $G$. First, let $c$ be a proper $(n-2)$-edge-coloring of $H$. For each vertex $x \in V(H)$, since $\operatorname{deg}_{H} x \leq n-3$, there is $a_{x} \in[n-2]$ such that $a_{x}$ is not assigned to any edge incident with $x$. Define $c(v x)=a_{x}$. Thus, the set $E_{x}$ of edges incident with $x$ is a rainbow set for each $x \in V(H)$. Let $u$ and $w$ be two distinct vertices of $G$. Then at least one of $u$ and $w$ belongs to $H$, say $u \in V(H)$. Since $E_{u}$ separates $u$ and $w$, it follows that $c$ is a rainbow disconnection coloring of $G$ using $n-2$ colors. Hence, $\operatorname{rd}(G) \leq n-2$.

Case 2. No vertex of $G$ has degree $n-1$. Therefore $\Delta(G) \leq n-2$. If $\Delta(G) \leq n-3$, then $\operatorname{rd}(G) \leq \chi^{\prime}(G) \leq n-2$ by Proposition 2.2. Thus, we may assume that $\Delta(G)=n-2$. Suppose first that $G$ is not $(n-2)$-regular. We claim that $G$ is a connected spanning subgraph of some graph $G^{*}$ of order $n$ having exactly one vertex of degree $n-1$. Let $u$ be a vertex of degree $k \leq n-3$ in $G$. Let $N(u)$ be the neighborhood of $u$ and $W=V(G)-N[u]$, where $N[u]=N(u) \cup\{u\}$ is the closed neighborhood of $u$. Then $|N(u)|=k$ and $|W|=n-k-1 \geq 2$. If $W$ contains a vertex $v$ of degree $n-2$ in $G$, then $v$ is the only vertex of degree $n-1$ in $G^{*}=G+u v$. If no vertex in $W$ has degree $n-2$ in $G$, then let $G^{*}$ be the graph obtained from $G$ by joining $u$ to each vertex in $W$. In this case, $u$ is the only vertex of degree $n-1$ in $G^{*}$. It then follows by Case 1 that $\operatorname{rd}\left(G^{*}\right) \leq n-2$. Since $G$ is a connected spanning subgraph of $G^{*}$, it follows by Proposition 2.6 that $\operatorname{rd}(G) \leq \operatorname{rd}\left(G^{*}\right) \leq n-2$. Finally, suppose that $G$ is $(n-2)$-regular. Thus, $G$ is 1-factorable and so $\chi^{\prime}(G)=\Delta(G)=n-2$. Therefore, $\operatorname{rd}(G) \leq \chi^{\prime}(G)=n-2$ by Proposition 2.2.

\section{Rainbow Disconnection in Grids and Prisms}

We now determine the rainbow disconnection numbers of graphs belonging to one of two well-known classes formed by Cartesian products. The Cartesian product $G \square H$ of two vertex-disjoint graphs $G$ and $H$ is the graph with vertex set $V(G) \times V(H)$, where $(u, v)$ is adjacent to $(w, x)$ in $G \square H$ if and only if either $u=w$ and $v x \in E(H)$ or $u w \in E(G)$ and $v=x$. We consider the $m \times n$ grid graph $G_{m, n}=P_{m} \square P_{n}$, which consists of $m$ horizontal paths $P_{n}$ and $n$ vertical paths $P_{m}$.

Theorem 4.1. The rainbow disconnection numbers of the grid graphs $G_{m, n}$ are as follows:

(i) for all $n \geq 2, \operatorname{rd}\left(G_{1, n}\right)=\operatorname{rd}\left(P_{n}\right)=1$,

(ii) for all $n \geq 3, \operatorname{rd}\left(G_{2, n}\right)=3$,

(iii) for all $n \geq 4, \operatorname{rd}\left(G_{3, n}\right)=3$,

(iv) for all $4 \leq m \leq n, \operatorname{rd}\left(G_{m, n}\right)=4$. 
Proof. (i) That $\operatorname{rd}\left(G_{1, n}\right)=\operatorname{rd}\left(P_{n}\right)=1$ for $n \geq 2$ is a consequence of Proposition 3.1.

For the remainder of the proof, we consider the vertices of $G_{m, n}$ as a matrix, letting $x_{i, j}$ denote the vertex in row $i$ and column $j$, where $1 \leq i \leq m$ and $1 \leq j \leq n$.

(ii) For the graph $G_{2, n}, n \geq 3, \Delta\left(G_{2, n}\right)=3$. First, we define an edge-coloring $c$ of $G_{2, n}$. It is convenient to use the elements of the set $\mathbf{Z}_{3}$ of integer modulo 3 as colors here. Define the edge-coloring $c: E\left(G_{2, n}\right) \rightarrow \mathbf{Z}_{3}$ by

$\star c\left(x_{i, j} x_{i, j+1}\right)=i+j+1$ for $1 \leq i \leq 2$ and $1 \leq j \leq n-1$;

$\star c\left(x_{1, j} x_{2, j}\right)=j$ for $1 \leq j \leq n$.

Next, we show that $c$ is a rainbow disconnection coloring of $G_{2, n}$. Let $u$ and $v$ be any two vertices of $G_{2, n}$. If $u$ and $v$ belong to two different columns, then there exist two parallel edges joining vertices in the same two columns whose removal separates $u$ and $v$. Each such set of two edges is a $u-v$ rainbow cut. Next, suppose that $u$ and $v$ belong to the same column. Then, without loss of generality, $u$ belongs to the first row and $v$ belongs to the second row. Then $u$ and $v$ both have degree 2 or both have degree 3 . Therefore, the edges incident with $u$ form a rainbow cut, and so, $\operatorname{rd}\left(G_{2, n}\right) \leq 3$.

On the other hand, $\lambda(u, v)=2$ if $u$ and $v$ are two vertices of $G_{2, n}$ belonging to the same row, or different rows and columns or are two vertices of degree 2 belonging to the same column; while $\lambda(u, v)=3$ if $u$ and $v$ are (adjacent) vertices of degree 3 belonging to the same column. It then follows by Proposition 2.2 that $3=\lambda^{+}\left(G_{2, n}\right) \leq \operatorname{rd}\left(G_{2, n}\right)$, and so $\operatorname{rd}\left(G_{2, n}\right)=3$.

(iii) As with $G_{2, n}$, we define an edge-coloring $c$ of $G_{3, n}$. Again we use the elements of the set $\mathbf{Z}_{3}$ of integer modulo 3 as colors here. Define the edge-coloring $c: E\left(G_{3, n}\right) \rightarrow \mathbf{Z}_{3}$ by

$\star c\left(x_{i, j} x_{i, j+1}\right)=i+j+1$ for $1 \leq i \leq 3$ and $1 \leq j \leq n-1$;

$\star c\left(x_{1, j} x_{2, j}\right)=j$ for $1 \leq j \leq n$;

$\star c\left(x_{2, j} x_{3, j}\right)=j+2$ for $1 \leq j \leq n$.

Next, we show that $c$ is a rainbow disconnection coloring of $G_{3, n}$. Let $u$ and $v$ be any two vertices of $G_{3, n}$. If $u$ and $v$ belong to two different columns, then there exist three parallel edges joining vertices in the same two columns whose removal separates $u$ and $v$. Each such set of three edges is a $u-v$ rainbow cut. Next, suppose that $u$ and $v$ belong to the same column. Then at least one of $u$ and $v$ belongs to the top or bottom row, say $u$ is such a vertex, which has degree 2 or 3 . Then the edges incident with $u$ is a $u-v$ rainbow cut. Thus, $\operatorname{rd}\left(G_{3, n}\right) \leq 3$.

On the other hand, for any two adjacent vertices $u$ and $v$ of degree 4 in $G_{3, n}$ (necessarily in the middle row), $\lambda^{+}(u, v)=3$. Thus, by Proposition 2.2, $3 \leq \lambda^{+}\left(G_{3, n}\right) \leq \operatorname{rd}\left(G_{3, n}\right) \leq 3$ and so $\operatorname{rd}\left(G_{3, n}\right)=3$. 
(iv) Finally, we consider $G_{m, n}$ for $4 \leq m \leq n$. Since there are four pairwise edge-disjoint $u-v$ paths in $G_{m, n}$ for every two vertices $u$ and $v$ of degree 4 , it follows by Theorem 2.1 that $\lambda(u, v)=4$. For any other pair $u, v$ of vertices of $G_{m, n}$, it follows that $\lambda(u, v) \leq 3$. By Proposition 2.2 then, $4=\lambda^{+}\left(G_{m, n}\right) \leq$ $\operatorname{rd}\left(G_{m, n}\right)$. On the other hand, since $G_{m, n}$ is bipartite, $\chi^{\prime}\left(G_{m, n}\right)=\Delta\left(G_{m, n}\right)=4$. Again, by Proposition $2.2, \operatorname{rd}\left(G_{m, n}\right) \leq 4$ and so $\operatorname{rd}\left(G_{4, n}\right)=4$.

Next we determine the rainbow disconnection number of prisms, namely those graphs of the type $G \square K_{2}$ for some graph $G$.

Proposition 4.2. If $G$ is a nontrivial connected graph, then

$$
\operatorname{rd}\left(G \square K_{2}\right)=\Delta(G)+1 .
$$

Proof. Let $G$ and $G^{\prime}$ be the two copies of $G$ in the prism $G \square K_{2}$, and for each $v \in V(G)$, let $v^{\prime}$ be its corresponding vertex in $G^{\prime}$. We first show that $G \square K_{2}$ has a proper edge-coloring using $\Delta\left(G \square K_{2}\right)=\Delta(G)+1$ colors, that is, $\chi^{\prime}\left(G \square K_{2}\right)=\Delta(G)+1$. Let $C$ be a proper edge-coloring of $G$ using $\chi^{\prime}(G)$ colors. Color the edges of $G$ and $G^{\prime}$ using $C$, that is, $G$ and $G^{\prime}$ have an identical edgecoloring $C$. By Vizing's Theorem, $\Delta(G) \leq \chi^{\prime}(G) \leq \Delta(G)+1$. First assume that $\chi^{\prime}(G)=\Delta(G)$. Then assigning the color $\Delta(G)+1$ to each edge $v v^{\prime}$ for every $v \in$ $V(G)$ gives a proper edge-coloring of $G \square K_{2}$ with $\Delta(G)+1$ colors. Next assume that $\chi^{\prime}(G)=\Delta(G)+1$. Then for each $v \in V(G)$, at least one of the $\Delta(G)+1$ colors is missing from the colors of the edges incident to $v$. Let $c_{v}$ be one such missing color. Note that $c_{v}$ is also missing from the colors of the edges incident to $v^{\prime}$ in $G^{\prime}$ because $G$ and $G^{\prime}$ have the identical colorings. Hence, assigning $c_{v}$ to $v v^{\prime}$ for each $v \in V(G)$ yields a proper edge-coloring of $G \square K_{2}$ having $\Delta(G)+1$ colors. By Proposition 2.2, it follows that $\operatorname{rd}\left(G \square K_{2}\right) \leq \Delta(G)+1$.

To establish the lower bound, let $u$ be a vertex of $G$ with $\operatorname{deg} u=\Delta(G)=\Delta$. In $G \square K_{2}$, there exist $\Delta+1$ edge-disjoint $u-u^{\prime}$ paths, one of which is the edge $u u^{\prime}$ and the remaining $\Delta$ of which have the form $\left(u, w, w^{\prime}, u^{\prime}\right)$, where $w \in V(G)$ and $w^{\prime}$ is the corresponding vertex of $w$ in $G^{\prime}$. It again follows by Proposition 2.2 that $\operatorname{rd}\left(G \square K_{2}\right) \geq \lambda^{+}\left(G \square K_{2}\right) \geq \Delta(G)+1$.

Complementary products were introduced in [4] as a generalization of Cartesian products. We consider a subfamily of complementary products, namely, complementary prisms. For a graph $G=(V, E)$, the complementary prism, denoted $G \bar{G}$, is formed from the disjoint union of $G$ and its complement $\bar{G}$ by adding a perfect matching between corresponding vertices of $G$ and $\bar{G}$. For each $v \in V(G)$, let $\bar{v}$ denote the vertex in $\bar{G}$ corresponding to $v$. Formally, the graph $G \bar{G}$ is formed from $G \cup \bar{G}$ by adding the edge $v \bar{v}$ for every $v \in V(G)$. We note that complementary prisms are a generalization of the Petersen graph. In particular, the Petersen graph is the complementary prism $C_{5} \bar{C}_{5}$. For another example of a complementary prism, the corona $K_{n} \circ K_{1}$ is the complementary prism $K_{n} \bar{K}_{n}$. 
We refer to the complementary prism $G \bar{G}$ as a copy of $G$ and a copy of $\bar{G}$ with a perfect matching between corresponding vertices. For a set $S \subseteq V(G)$, let $\bar{S}$ denote the corresponding set of vertices in $V(\bar{G})$. We note that $G \bar{G}$ is isomorphic to $\bar{G} G$.

Since $\Delta(G \bar{G})=\max \{\Delta(G), \Delta(\bar{G})\}+1$, Proposition 2.2 implies that $\operatorname{rd}(G \bar{G}) \leq$ $\max \{\Delta(G), \Delta(\bar{G})\}+2$. This bound is sharp for the Petersen graph $P=C_{5} \bar{C}_{5}$ since by Proposition 2.5, $\operatorname{rd}(P)=4=\Delta\left(C_{5}\right)+2$. On the other hand, for the complementary prisms $K_{n} \bar{K}_{n}$, Corollary 2.11 and Proposition 3.5 imply that $\operatorname{rd}\left(K_{n} \bar{K}_{n}\right)=\operatorname{rd}\left(K_{n}\right)=n-1=\Delta\left(K_{n}\right)<\max \left\{\Delta\left(K_{n}\right), \Delta\left(\bar{K}_{n}\right\}+2=n+1\right.$. Our next result shows that for graphs $G$ with sufficiently large girth, $\operatorname{rd}(G \bar{G})$ is strictly greater than the maximum degree of $G$.

Proposition 4.3. If $G$ is a graph of order $n$, maximum degree $\Delta(G)<n-1$, and girth at least five, then

$$
\Delta(G)+1 \leq \operatorname{rd}(G \bar{G}) .
$$

Proof. Consider a vertex $u$ in $G$ such that $\operatorname{deg}_{G} \mathrm{u}=\Delta(\mathrm{G})$. Let $A=N_{G}(u)$ and $B=V-N_{G}[u]$. Thus, in $G \bar{G}, N(\bar{u})=\bar{B} \cup\{u\}$. Note that since $n-1>\Delta(G)$, it follows that $\bar{B} \neq \emptyset$.

We claim there are $\Delta(G)+1$ edge-disjoint $u-\bar{b}$ paths, where $\bar{b} \in \bar{B}$. To see this note that one such path is $(u, \bar{u}, \bar{b})$. Next consider the $u-\bar{b}$ paths containing a vertex $a \in A$. If $a$ is not adjacent to $b$ in $G$, then $\bar{a}$ is adjacent to $\bar{b}$ in $\bar{G}$ and $(u, a, \bar{a}, \bar{b})$ is a $u-\bar{b}$ path. If $a b \in E(G)$, then $(u, a, b, \bar{b})$ is a $u-\bar{b}$ path. Moreover, since $g(G) \geq 5$, at most one vertex in $A$ is adjacent to $b$, else a 4-cycle is formed. In any case, the collection of these $|A|+1=\Delta(G)+1$ paths are edge-disjoint. Hence, by Proposition 2.2, it follows that $\operatorname{rd}(G \bar{G}) \geq \lambda^{+}(G \bar{G}) \geq \Delta(G)+1$.

For an example of a complementary prism attaining the lower bound, let $G$ be the graph formed from a 5-cycle by attaching a leaf $x$ to a vertex $v$ of the cycle. Then, $\Delta(G)=3$. We show that $\operatorname{rd}(G \bar{G})=4$. First note that the Petersen graph $P$ is a proper subgraph of $G \bar{G}$, and by Propositions 2.5 and 2.6, $\operatorname{rd}(G \bar{G}) \geq \operatorname{rd}(P)=4$. Furthermore, there is a proper edge-coloring $c$ of $P$ using four colors such that three colors are used to color $C_{5}$ and $\bar{C}_{5}$ and the fourth color is used on the matching edges. Thus, we may assume, without loss of generality, that $v$ is incident to the edges colored 1 and 2 in $G$ and that $v \bar{v}$ is assigned color 4 . We extend $c$ to a rainbow disconnection coloring of $G \bar{G}$ as follows: let $c(v x)=3$, $c(x \bar{x})=4$, and $c(\overline{x u})$ be the color missing from the edges incident to $\bar{u}$ for each $\bar{u}$ adjacent to $\bar{x}$ in $\bar{G}$. Consider two arbitrary vertices of $G \bar{G}$. At least one of the vertices, say $u$, is not $\bar{x}$. Thus, the edges incident with $u$ are a rainbow cut separating the two vertices. Since every such vertex $u$ has degree at most four, $\operatorname{rd}(G \bar{G}) \leq 4$, and so, $\operatorname{rd}(G \bar{G})=4$. 


\section{Extremal Problems}

In this section, we investigate the following problem:

For a given pair $k, n$ of positive integers with $k \leq n-1$, what are the minimum possible size and maximum possible size of a connected graph $G$ of order $n$ such that the rainbow disconnection number of $G$ is $k$ ?

We have seen in Proposition 3.1 that the only connected graphs of order $n$ having rainbow disconnection number 1 are the trees of order $n$. That is, the connected graphs of order $n$ having rainbow disconnection number 1 have size $n-1$. We have also seen in Theorem 3.4 that the minimum size of a connected graph of order $n \geq 3$ having rainbow disconnection number 2 is $n$. Furthermore, we have seen in Theorem 3.6 that the minimum size of a connected graph of order $n \geq 2$ having rainbow disconnection number $n-1$ is $2 n-3$. In fact, these are special cases of a more general result. In order to show this, we first present a lemma.

Lemma 5.1. Let $H$ be a connected graph of order $n$ that is not complete and let $x$ and $y$ be two nonadjacent vertices of $H$. Then $\operatorname{rd}(H+x y) \leq \operatorname{rd}(H)+1$.

Proof. Suppose that $\operatorname{rd}(H)=k$ for some positive integer $k$ and let $c_{0}$ be a rainbow disconnection coloring of $H$ using the colors $1,2, \ldots, k$. Extend the coloring $c_{0}$ to the edge-coloring $c$ of $H+x y$ by assigning the color $k+1$ to the edge $x y$. Let $u$ and $v$ be two vertices of $H$ and let $R$ be a $u-v$ rainbow cut in $H$. Then $R \cup\{x y\}$ is a $u-v$ rainbow cut in $H+x y$. Hence, $c$ is a rainbow disconnection $(k+1)$-coloring of $H+x y$. Therefore, $\operatorname{rd}(H+x y) \leq k+1=\operatorname{rd}(H)+1$.

Theorem 5.2. For integers $k$ and $n$ with $1 \leq k \leq n-1$, the minimum size of a connected graph of order $n$ having rainbow disconnection number $k$ is $n+k-2$.

Proof. By Proposition 3.5, the result is true for $k=n-1$. Hence, we may assume that $1 \leq k \leq n-2$. First, we show that if the size of a connected graph $G$ of order $n$ is $n+k-2$, then $\operatorname{rd}(G) \leq k$. We proceed by induction on $k$. We have seen that the result is true for $k=1,2$ by Proposition 3.1 and Theorem 3.4. Suppose that if the size of a connected graph $H$ of order $n$ is $n+k-2$ for some integer $k$ with $2 \leq k \leq n-3$, then $\operatorname{rd}(H) \leq k$. Let $G$ be a connected graph of order $n$ and size $n+(k+1)-2=n+k-1$. We show that $\operatorname{rd}(G) \leq k+1$. Since $G$ is not a tree, there is an edge $e$ such that $H=G-e$ is a connected spanning subgraph of $G$. Since the size of $H$ is $n+k-2$, it follows by induction hypothesis that $\operatorname{rd}(H) \leq k$. Hence, $\operatorname{rd}(G)=\operatorname{rc}(H+e) \leq k+1$ by Lemma 5.1. Therefore, the minimum possible size for a connected graph $G$ of order $n$ to have $\operatorname{rd}(G)=k$ is $n+k-2$.

It remains to show that for each pair $k, n$ of integers with $1 \leq k \leq n-1$ there is a connected graph $G$ of order $n$ and size $n+k-2$ such that $\operatorname{rd}(G)=k$. Since this 
is true for $k=1,2, n-1$, we now assume that $3 \leq k \leq n-2$. Let $H=K_{2, k}$ with partite set $U=\left\{u_{1}, u_{2}\right\}$ and $W=\left\{w_{1}, w_{2}, \ldots, w_{k}\right\}$. Now, let $G$ be the graph of order $n$ and size $n+k-2$ obtained from $H$ by subdividing the edge $u_{1} w_{1}$ a total of $n-k-2$ times, producing the path $P=\left(u_{1}, v_{1}, v_{2}, \ldots, v_{n-k-2}, w_{1}\right)$ in $G$. Since $\chi^{\prime}(H)=k$, there is a proper edge-coloring $c_{H}$ of $H$ using the colors $1,2, \ldots, k$. We may assume that $c\left(u_{1} w_{1}\right)=1$ and $c\left(u_{2} w_{1}\right)=2$. Next, we extend the coloring $c_{H}$ to a proper edge-coloring $c_{G}$ of $G$ using the colors $1,2, \ldots, k$ by defining $c_{G}\left(u_{1} v_{1}\right)=1$ and alternating the colors of the edges of $P$ with 3 and 1 thereafter. Hence, $\chi^{\prime}(G)=k$ and so $\operatorname{rd}(G) \leq \chi^{\prime}(G)=k$ by Proposition 2.2. Furthermore, since $\lambda\left(u_{1}, u_{2}\right)=k$ and $\lambda(x, y)=2$ for all other pairs $x, y$ of vertices of $G$, it follows that $\lambda^{+}(G)=k$. Again, by Proposition $2.2, \operatorname{rd}(G) \geq \lambda^{+}(G)=k$ and so $\operatorname{rd}(G)=k$.

For given integers $k$ and $n$ with $1 \leq k \leq n-1$, we have determined the minimum size of a connected graph $G$ of order $n$ with $\operatorname{rd}(G)=k$. So, this brings up the question of determining the maximum size of a connected graph $G$ of order $n$ with $\operatorname{rd}(G)=k$. Of course, we know this size when $k=1$; it is $n-1$. Also, we know this size when $k=n-1$; it is $\left(\begin{array}{l}n \\ 2\end{array}\right)$. For odd integers $n$, we have the following conjecture.

Conjecture 5.3. Let $k$ and $n$ be integers with $1 \leq k \leq n-1$ and $n \geq 5$ is odd. Then the maximum size of a connected graph $G$ of order $n$ with $\operatorname{rd}(G)=k$ is $\frac{(k+1)(n-1)}{2}$.

Notice that when $k=1$, then $\frac{(k+1)(n-1)}{2}=n-1$ and when $k=n-1$, then $\frac{(k+1)(n-1)}{2}=\left(\begin{array}{l}n \\ 2\end{array}\right)$. Also, when $k=2$, then $\frac{(k+1)(n-1)}{2}=\frac{3 n-3}{2}$. This is the size of the so-called friendship graph $\left(\frac{k-1}{2}\right) K_{2} \vee K_{1}$ of order $n$ (every two vertices has a unique friend). Since each block of a friendship graph is a triangle, it follows by Theorem 3.4 that each such graph has rainbow disconnection number 2 .

For given integers $k$ and $n$ with $1 \leq k \leq n-1$ and $n \geq 5$ is odd, let $H_{k}$ be a $(k-1)$-regular graph of order $n-1$. Since $n-1$ is even, such graphs $H_{k}$ exist. Now, let $G_{k}=H_{k} \vee K_{1}$ be the join of $H_{k}$ and $K_{1}$. Thus, $G_{k}$ is a connected graph of order $n$ having one vertex of degree $n-1$ and $n-1$ vertices of degree $k$. The size $m$ of $G_{k}$ satisfies the equation:

$$
2 m=(n-1)+(n-1) k=(k+1)(n-1)
$$

and so $m=\frac{(k+1)(n-1)}{2}$. The graph $H_{k}$ can be selected so that it is 1-factorable and so $\chi^{\prime}\left(H_{k}\right)=k-1$. If a proper $(k-1)$-edge-coloring of $H_{k}$ is given using the colors $1,2, \ldots, k-1$, and every edge incident with the vertex of $G_{k}$ of degree $n-1$ is assigned the color $k$, then the edges incident with each vertex of degree $k$ are properly colored with $k$ colors. For any two vertices $u$ and $v$ of $G_{k}$, at least one of 
$u$ and $v$ has degree $k$ in $G_{k}$, say $\operatorname{deg}_{G_{k}} u=k$. Then the set of edges incident with $u$ is a $u-v$ rainbow cut in $H$. Since this is a rainbow disconnection $k$-coloring of $G$, it follows that $\operatorname{rd}\left(G_{k}\right) \leq k$. It is reasonable to conjecture that $\operatorname{rd}\left(G_{k}\right)=k$.

We would still be left with the question of whether every graph $H$ of order $n$ and size $\frac{(k+1)(n-1)}{2}+1$ must have $\operatorname{rd}(H)>k$. Certainly, every such graph $H$ must contain at least two vertices whose degrees exceed $k$.

\section{Acknowledgment}

We greatly appreciate the valuable suggestions made by two anonymous referees, which resulted in an improved paper.

\section{REFERENCES}

[1] G. Chartrand, G.L. Johns, K.A. McKeon and P. Zhang, Rainbow connection in graphs, Math. Bohem. 133 (2008) 85-98.

[2] P. Elias, A. Feinstein and C.E. Shannon, A note on the maximum flow through a network, IRE Trans. Inform. Theory, IT 2 (1956) 117-119. doi:10.1109/TIT.1956.1056816

[3] L.R. Ford Jr. and D.R. Fulkerson, Maximal flow through a network, Canad. J. Math. 8 (1956) 399-404. doi:10.4153/CJM-1956-045-5

[4] T.W. Haynes, M.A. Henning, P.J. Slater and L.C. van der Merwe, The complementary product of two graphs, Bull. Inst. Combin. Appl. 51 (2007) 21-30.

[5] X.L. Li, Y. Shi and Y.F. Sun, Rainbow connections of graphs: A survey, Graphs Combin. 29 (2013) 1-38. doi:10.1007/s00373-012-1243-2

[6] X.L. Li and Y.F. Sun, Rainbow Connections of Graphs (Springer, Boston, MA, 2012).

doi:10.1007/978-1-4614-3119-0

[7] V.G. Vizing, On an estimate of the chromatic class of a p-graph, Diskret. Anal. 3 (1964) 25-30, in Russian.

[8] H. Whitney, Congruent graphs and the connectivity of graphs, Amer. J. Math. 54 (1932) 150-168.

doi:10.2307/2371086

Received 12 December 2016

Revised 12 April 2017

Accepted 12 April 2017 